\title{
Motion Feasibility of Multi-Agent Formations
}

\author{
Paulo Tabuada, Member, IEEE, George J. Pappas, Member, IEEE, and Pedro Lima, Member, IEEE
}

\begin{abstract}
Formations of multi-agent systems, such as mobile robots, satellites and aircraft, require individual agents to satisfy their kinematic equations while constantly maintaining interagent constraints. In this paper, we develop a systematic framework for studying formation motion feasibility of multi-agent systems. In particular, we consider formations wherein all the agents cooperate to enforce the formation. We determine algebraic conditions that guarantee formation feasibility given the individual agent kinematics. Our framework also enables us to obtain lower dimensional control systems describing the group kinematics while maintaining all formation constraints.
\end{abstract}

Index Terms_Formations, motion feasibility, multi-agent.

\section{INTRODUCTION}

A DVANCES in communication and computation have enabled the distributed control of multi-agent systems. This philosophy has resulted in next generation automated highway systems [21], coordination of aircraft in future air traffic management systems [20], as well as formation flying aircraft, satellites, and multiple mobile robots [2], [3], [8]. The control of multi-agent systems is greatly simplified when the agent's mission can be executed by means of a formation. In several applications, maintaining a formation is even fundamental as in multiple aircraft where the formation is used to explore aerodynamic effects [5] or in robotic exploration of large areas with restricted sensor capabilities [7].

The various approaches to formation control of a group of agents can roughly be divided into three categories: behavior-based, leader-follower, and rigid-body type formations. Behavior based approaches [2] start by designing simple and intuitive behaviors or motion primitives for each individual agent. Then, by a weighted sum of this simple primitives more complex motion patterns are generated through the interaction of several agents. Although this approach is characterized by being difficult to analyze in a rigorous and formal way, some of these simple schemes have already proven to be stable and convergent [12]. In leader-follower approaches [13], [19],

Manuscript received January 8, 2004; revised June 1, 2004. This paper was recommended for publication by Associate Editor S. Ma and Editor F. Park upon evaluation of the reviewers' comments. The work of P. Tabuada was supported in part by Fundação para a Ciência e Tecnologia under Grant PRAXIS XXI/BD/18149/98. The work of G. J. Pappas was supported in part by the Defense Advanced Research Projects Agency/AFRL Software-Enabled Control under Grant F33615-01-C-1848.

P. Tabuada is with the Department of Electrical Engineering, University of Notre Dame, Notre Dame, IN 46556 USA (e-mail: ptabuada@ nd.edu).

G. J. Pappas is with the Department of Electrical and Systems Engineering, University of Pennsylvania, Philadelphia, PA 19104 USA (e-mail: pappasg@ seas.upenn.edu).

P. Lima is with Instituto de Sistemas e Robótica, and Instituto Superior Técnico, 1049-001 Lisboa, Portugal (e-mail: pal@isr.ist.utl.pt).

Digital Object Identifier 10.1109/TRO.2004.839224 one or more agents are designated as leaders and are responsible for guiding the formation. The remaining agents are required to follow the leader with a predefined offset. Finally, in Rigid-Body type formations [4], [10], [14], [15] the distance between the agents configurations (usually their positions) is required to be constant during all formation motions.

Many fundamental questions remain unanswered in this recent area of formation control. The control of a formation requires individual agents to satisfy their kinematics while constantly satisfying interagent constraints. In typical leader-follower formations, the leader has the responsibility of guiding the group, while the followers have the responsibility of maintaining the interagent formation. Distributing the group control tasks to individual agents must be compatible with the control and sensing capabilities of the individual agents. As the interagent dependencies get more complicated, a systematic framework for controlling formations is vital.

In this paper, we propose a framework to determine motion feasibility of multi-agent formations. Formations are modeled using formations graphs which are graphs whose nodes capture the individual agent kinematics, and whose edges represent interagent constraints that must be satisfied. A similar modeling framework has been proposed in [9], and in [15] and [19], graph theoretical methods are used to analyze formation stability properties. Similar problems arise in the study of formation rigidity properties [10]. This class of systems is rich enough to capture holonomic, nonholonomic, or underactuated agents.

In this paper, we focus on the feasibility problem: Given the kinematics of several agents along with interagent constraints, determine whether there exist nontrivial agent trajectories that maintain the constrains. We obtain algebraic conditions that determine formation motion feasibility. A related problem is to determine formation rigidity and in [10] it is shown how rigidity can be determined by the analysis of a rigidity matrix. Such matrix is a representation of the codistribution $\Omega$ introduced in Section IV. However, we focus on motion feasibility for a larger class of formations including, but not restricted to rigid formations.

When a formation has feasible motions, the formation control abstraction problem is then considered: Given a formation with feasible motions, obtain a lower dimensional control system that maintains formation along its trajectories. Such control system allows to control the formation as a single entity, therefore being well suited for higher levels of control. A preliminary version of the results presented in this paper appeared in [17] and [18].

\section{Mathematical PRELIMINARIES}

In this section, we introduce some usual notation in control theory [11]. A function $f: \mathbb{R}^{n} \rightarrow \mathbb{R}^{m}$ is said smooth if it is infinitely differentiable. For a given smooth function $g: \mathbb{R}^{n} \rightarrow \mathbb{R}$ 
we denote by $\mathbf{d} g$ the row vector containing the partial derivatives of $g$, that is

$$
\mathbf{d} g=\left[\frac{\partial g}{\partial x_{1}} \frac{\partial g}{\partial x_{2}} \ldots \frac{\partial g}{\partial x_{n}}\right] .
$$

A distribution $\Delta_{x}$ on $\mathbb{R}^{n}$ is an assignment of a linear subspace of $\mathbb{R}^{n}$ at each point $x \in \mathbb{R}^{n}$. The rank of a distribution at a point $x \in \mathbb{R}^{n}$ is the dimension of the subspace $\Delta_{x} \subseteq \mathbb{R}^{n}$. In this paper we will assume that all distributions have constant rank which implies that, locally, there exist vector fields $X_{1}, X_{2}, \ldots, X_{k}$ such that $\operatorname{span}\left\{X_{1}(x), X_{2}(x), \ldots, X_{k}(x)\right\}=\Delta_{x}$. We say that a vector field $X: \mathbb{R}^{n} \rightarrow \mathbb{R}^{n}$ belongs to a distribution $\Delta$ if $X(x) \in \Delta_{x} \forall x \in \mathbb{R}^{n}$. As distributions are given by the span of column vectors, codistributions are defined in terms of row vectors. We denote by $\mathbb{R}^{n *}$ the space of all row vectors $\alpha$ such that $\alpha^{T} \in \mathbb{R}^{n}$, where by $\alpha^{T}$ we denote the column vector obtained by transposing $\alpha$. We now define codistributions as assignments of linear subspaces of $\mathbb{R}^{n *}$. Given a distribution $\Delta$, there is a unique annihilating codistribution $\Delta^{\perp}$ defining $\Delta$. This codistribution is defined as

$$
\Delta^{\perp}=\left\{\alpha \in \mathbb{R}^{n *} \quad \mid \quad \alpha \cdot X=0 \quad \forall X \in \Delta\right\} .
$$

Conversely, a codistribution $\Delta^{\perp}$ defines a unique distribution $\Delta$ given by the set of all vector fields $X$ such that $\Delta^{\perp} \cdot X=0$, that is, $\alpha \cdot X=0$ for every $\alpha \in \Delta^{\perp}$. Given distributions $\Delta$ on $\mathbb{R}^{n}$ and $\Delta^{\prime}$ on $\mathbb{R}^{n^{\prime}}$ we define their direct sum $\Delta \oplus \Delta^{\prime}$ as the direct sum of the vector space $\Delta_{x_{1}}$ with the vector space $\Delta_{x_{2}}^{\prime}$ for every $\left(x_{1}, x_{2}\right) \in \mathbb{R}^{n} \times \mathbb{R}^{n^{\prime}}$.

In this paper, we shall restrict attention to drift free control systems. Such control systems can be represented by

$$
\dot{x}=\sum_{j=1}^{l} X_{j} u_{j}
$$

where $X_{j}$ are smooth vector fields on $\mathbb{R}^{n}$ and $u_{j}$ the control inputs. A trajectory of (3) is a smooth curve $x: I \rightarrow \mathbb{R}^{n}$ for which there exists another smooth curve $u: I \rightarrow \mathbb{R}^{l}$ such that (3) is satisfied for every $t$ in the open set $I$ contained in $\mathbb{R}$. Drift free control systems are equivalently described by the distribution

$$
\Delta_{x}=\operatorname{span}\left\{X_{1}(x), X_{2}(x), \ldots, X_{l}(x)\right\}
$$

capturing all possible directions of motion or by the codistribution $\Delta_{x}^{\perp}$. This class of control systems is general enough to capture underactuated as well as holonomic or nonholonomic systems.

Example 2.1: Consider, for example, a unicycle type robot. If we model its state space by $\mathbb{R}^{3}$ where a point is denoted by $(x, y, \theta)$ with $x$ and $y$ representing the robot's position and $\theta$ the robot's orientation, we can define its kinematics by

$$
\begin{aligned}
& \dot{x}=u_{1} \cos \theta \\
& \dot{y}=u_{1} \sin \theta \\
& \dot{\theta}=u_{2} .
\end{aligned}
$$

Introducing the vector fields

$$
X_{1}=\left[\begin{array}{c}
\cos \theta \\
\sin \theta \\
0
\end{array}\right] \quad X_{2}=\left[\begin{array}{l}
0 \\
0 \\
1
\end{array}\right]
$$

we can rewrite (5) as

$$
\dot{X}=[\dot{x} \dot{y} \dot{\theta}]^{T}=X_{1} u_{1}+X_{2} u_{2} .
$$

Equation (7) shows that all the possible directions of motion allowed by (5) are captured by the distribution

$$
\Delta=\operatorname{span}\left\{X_{1}, X_{2}\right\}
$$

or equivalently by its annihilating codistribution

$$
\Delta^{\perp}=\operatorname{span}\{\sin \theta \mathbf{d} x-\cos \theta \mathbf{d} y\}
$$

where in this case $\mathbf{d} x \cdot X=u_{1} \cos \theta$ and $\mathbf{d} y \cdot X=u_{1} \sin \theta$.

\section{FORMATION GRAPHS}

A formation of $r$ heterogeneous agents with states $x_{i}(t) \in$ $\mathbb{R}^{n_{i}}, i=1, \ldots, r$ and kinematics defined by codistributions $\Delta_{i}^{\perp}$ is modeled by a formation graph which completely describes individual agent kinematics and global interagent constrains.

Definition 3.1 (Formation Graph): A formation graph $F=$ $(V, E, C)$ consists of:

- a finite set $V$ of $r$ vertices, where $r$ is the number of agents in the formation. Each vertex $v_{i}$ is a codistribution $\Delta_{i}^{\perp}$ modeling agent $i$ kinematics;

- a binary and symmetric relation $E \subseteq V \times V$ representing a bond or link between the agents;

- a family of constraints $C$ indexed by the set $E, C=$

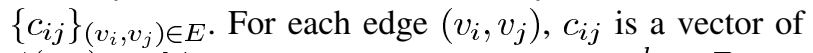
$\phi(i, j) \in \mathbb{N}$ smooth real valued functions $c_{i j}^{k}: \mathbb{R}^{n_{i}} \times$ $\mathbb{R}^{n_{j}} \rightarrow \mathbb{R}, k=1,2, \ldots, \phi(i, j)$ defining the formation constraints between agents $i$ and $j$. The constraint is enforced when $c_{i j}^{k}\left(x_{i}, x_{j}\right)=0$.

In Fig. 1, the formation graph used in Example 4.2 is represented graphically. The symmetry assumption on $E$ ensures that for each $\left(v_{i}, v_{j}\right) \in E,\left(v_{j}, v_{i}\right)$ also belongs to $E$ and in fact we identify $\left(v_{i}, v_{j}\right)$ with $\left(v_{i}, v_{j}\right)$ to guarantee that the same constraint is not accounted for twice. This allows to model constraints without a preferred sense of direction in which both agents are equally responsible for the constraint satisfaction. We also assume perfect communication between agents $v_{i}$ and $v_{j}$.

In this paper, we focus on the motion feasibility problem, more precisely

Problem 3.2 (Motion Feasibility): Given a formation graph $F=(V, E, C)$ determine whether there are nontrivial trajectories $x_{i}(t)$ of all agent kinematics (3) that maintain the constraints $c_{i j}^{k}$ for all $\left(v_{i}, v_{j}\right) \in E, k=1,2, \ldots, \phi(i, j)$ and $t \in I$.

When there are feasible motions, a new problem immediately emerges, the extraction of a formation control abstraction which characterizes the solution space of Problem 3.2: 


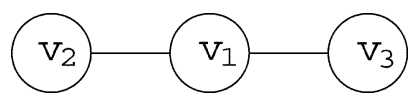

Fig. 1. Three agents formation.

Problem 3.3 (Abstraction): Given a formation graph $F=$ $(V, E, C)$ with feasible motions, obtain a lower dimensional control system that describes all feasible formation motions.

\section{UNDIRECTED FORMATIONS}

\section{A. Motion Feasibility}

In undirected formations each agent is equally responsible for maintaining constraints. Because of this property it will be useful to collect all agent kinematics and constraints on a single state space

$$
\mathbb{R}^{m}=\mathbb{R}^{n_{1}+n_{2}+\ldots+n_{n}}=\prod_{i=1}^{n} \mathbb{R}^{n_{i}}
$$

Given an element $x$ of $\mathbb{R}^{m}$ the canonical projection on the $i$ th agent $\pi_{i}: \mathbb{R}^{m} \rightarrow \mathbb{R}^{n_{i}}$ allow us to denote the state of the individual agents by $x_{i}=\pi_{i}(x)$. The formation kinematics is obtained by appending the individual kinematics through direct sum, that is

$$
\Delta^{\perp}=\oplus_{i=1}^{n} \Delta_{i}^{\perp}
$$

This new codistribution $\Delta^{\perp}$ on $\mathbb{R}^{m}$ describes the kinematics of all agents, however it does not model any interaction between them. This interaction will be induced by the formation constraints that we now lift to the group state space $\mathbb{R}^{m}$. Each constraint $c_{i j}^{k}$ linking agent $i$ to agent $j$ induces a constraint $\mathcal{C}_{i j}^{k}$ on $\mathbb{R}^{m}$ defined by

$$
\mathcal{C}_{i j}^{k}(x)=c_{i j}^{k}\left(\pi_{i}(x), \pi_{j}(x)\right) .
$$

All of these constraints can now be grouped in a single map from $\mathbb{R}^{m}$ to $\mathbb{R}^{d}$ with $d=\sum_{\left(v_{i}, v_{j}\right) \in E} \phi(i, j)$. This constraint map $\mathcal{C}$ is obtained by stacking all individual constraints as follows:

$$
\mathcal{C}=\left[\mathcal{C}_{1}^{1} \mathcal{C}_{1}^{2} \ldots \mathcal{C}_{1}^{\phi(1)} \mathcal{C}_{2}^{1} \mathcal{C}_{2}^{2} \ldots \mathcal{C}_{2}^{\phi(2)} \ldots \mathcal{C}_{q}^{1} \mathcal{C}_{q}^{2} \ldots \mathcal{C}_{q}^{\phi(q)}\right]^{T}
$$

where we have considered an enumeration $\{1,2, \ldots, q\}$ of the edge set $E$. Without loss of generality ${ }^{1}$ we assume that $\mathcal{C}$ has constant rank on a neighborhood of $\mathbf{0}$, consequently the set $\mathcal{C}^{-1}(\mathbf{0})=\left\{x \in \mathbb{R}^{m} \mid \mathcal{C}(x)=\mathbf{0}\right\}$ defines a submanifold $N$ of $\mathbb{R}^{m}$. This manifold $N$ characterizes the interaction between the agents since the state variables of each agent are required to live on this submanifold. Motion feasibility requires that the constraints are satisfied along the formation trajectories, that is, that the submanifold $N$ is invariant under $\Delta$ trajectories

$$
\left.\frac{d}{d t} \mathcal{C}_{i j}^{k}\right|_{t=0}=L_{X} \mathcal{C}_{i j}^{k}=\mathbf{d} \mathcal{C}_{i j}^{k} \cdot X=0
$$

${ }^{1}$ Since we can use Sard's theorem [1] on the map $\mathcal{C}$. This local rank assumption ensures that $\mathcal{C}$ is a subimmersion and therefore $\mathcal{C}^{-1}(\mathbf{0})$ is a submanifold of $\mathbb{R}^{m}$ [1]. Note that although the map $\mathcal{C}$ depends on the chosen enumeration, the submanifold it defines does not. for every $X \in \Delta,\left(v_{i}, v_{j}\right) \in E, k \in\{1,2, \ldots, \phi(i, j)\}$, and where $L_{X} \mathcal{C}_{i j}^{k}$ denotes the Lie derivative of $\mathcal{C}_{i j}^{k}$ with respect to $X$. We now capture all the constraints $\mathcal{C}_{i j}^{k}$ in a single codistribution

$$
\begin{aligned}
& \mathrm{d} \mathcal{C}=\operatorname{span}\left\{\mathbf{d} \mathcal{C}_{1}^{1}, \mathbf{d} \mathcal{C}_{1}^{2}, \ldots, \mathbf{d} \mathcal{C}_{1}^{\phi(1)}\right. \\
& \mathbf{d} \mathcal{C}_{2}^{1}, \mathbf{d} \mathcal{C}_{2}^{2}, \ldots, \mathbf{d} \mathcal{C}_{2}^{\phi(2)} \\
& \left.\mathbf{d} \mathcal{C}_{1}^{m}, \mathbf{d} \mathcal{C}_{1}^{m}, \ldots, \mathbf{d} \mathcal{C}_{m}^{\phi(m)}\right\}
\end{aligned}
$$

and see that a vector field $X$ satisfies (13) iff $\alpha \cdot X=0$ for every $\alpha \in \mathbf{d} \mathcal{C}$. This we shall denote ${ }^{2}$ by

$$
\mathbf{d} \mathcal{C} \cdot X=0 \text {. }
$$

Vector fields $X$ satisfying $\Delta^{\perp} \cdot X=0$ represent directions of motion respecting the individual agent kinematics, while vector fields $X$ satisfying $\mathrm{d} \mathcal{C} \cdot X=0$ represent directions of motion respecting the formation constraints. Therefore by merging both objects $^{3}$ into

$$
\Omega=\mathrm{d} \mathcal{C}+\Delta^{\perp}
$$

that is, by denoting by $\Omega$ the codistribution spanned by the union of a basis of $\mathbf{d} \mathcal{C}$ and a basis of $\Delta^{\perp}$, we can check for feasible motions in a single equation

$$
\Omega_{x} \cdot X(x)=0 \quad \forall x \in N
$$

Note that this equation only needs to hold for points belonging to $N$, since outside $N$ the agents are no longer in formation. The previous discussion leads to the following solution of Problem 3.2:

Theorem 4.1: An undirected formation has feasible motions iff (17) has nontrivial solutions, equivalently iff

$$
\operatorname{dim} \Omega_{x}<m
$$

for every $x \in N$.

The condition described in Theorem 4.1 can be tested by determining the rank of the matrix associated with distribution $\Omega$. Such computations can be performed in any symbolic computation package such as Mathematica. In many examples of interest, a basis for distribution $\Omega$ has less than $m$ elements which immediately allows to conclude that (18) holds. A solution of equation $\Omega \cdot X=0$ specifies the motion of each individual agent. When more than one independent solution exists, a change in the direction of a single agent may require that all other agents also change their actions to maintain formation. This shows that, in general, solutions are centralized and require interagent communication for their implementation.

Example 4.2: We now illustrate Theorem 4.1 in a simple example. Consider an undirected formation consisting of three unicycle type robots as displayed in Fig. 1. The kinematics of

\footnotetext{
${ }^{2}$ At the computational level, condition (15) is determined by constructing the matrix with the row vectors $\mathbf{d} \mathcal{C}_{1}^{1}, \mathbf{d} \mathcal{C}_{1}^{2}, \ldots, \mathbf{d} \mathcal{C}_{m}^{\phi(m)}$ appearing in the definition of $\mathrm{d} \mathcal{C}$ and multiplying such matrix by $X$.

${ }^{3}$ Computationally, the codistribution $\Omega$ is characterized by the matrix having as row vectors, the row vectors appearing in the matrices describing $\mathrm{d} \mathcal{C}$ and $\Delta^{\perp}$
} 
each agent is given by codistributions of the form (9). To completely specify the formation graph we need to define the interagent constraints. The constraint associated with the edge between agent 1 and agent 2 is defined by

$$
c_{12}=\left[\begin{array}{c}
x_{1}-x_{2}-\delta_{x} \\
y_{1}-y_{2}-\delta_{y} \\
\theta_{1}-\theta_{2}
\end{array}\right]
$$

where $\delta_{x}$ and $\delta_{y}$ are positive constants. There is also a constraint between agents 1 and 3 defined by

$$
c_{13}=\left[\frac{1}{2}\left(x_{1}-x_{3}\right)^{2}+\frac{1}{2}\left(y_{1}-y_{3}\right)^{2}+\frac{1}{2}\left(\theta_{1}-\theta_{3}\right)^{2}-\delta\right]
$$

with $\delta$ a positive constant. The constraint between agents 1 and 2 requires them to perform the same trajectories with an offset between their position coordinates given by $\delta_{x}$ and $\delta_{y}$ which we intuitively know to be possible. However, the constraint between agents 1 and 3 requires the distance between their positions to equal $\sqrt{2 \delta+(1 / 2)\left(\theta_{1}-\theta_{3}\right)^{2}}$. This is clearly a non intuitive constraint and no a priori answer can be given regarding feasibility. We will now study feasibility of this formation according to the methods developed so far. First, we compute $\Delta^{\perp}$

$$
\begin{aligned}
\Delta^{\perp}=\operatorname{span}\{ & -\sin \theta_{1} \mathbf{d} x_{1}+\cos \theta_{1} \mathbf{d} y_{1} \\
& -\sin \theta_{2} \mathbf{d} x_{2}+\cos \theta_{2} \mathbf{d} y_{2} \\
& \left.-\sin \theta_{3} \mathbf{d} x_{3}+\cos \theta_{3} \mathbf{d} y_{3}\right\} .
\end{aligned}
$$

Since $\mathcal{C}$ is given by

$$
\mathcal{C}=\left[\begin{array}{c}
x_{1}-x_{2}-\delta_{x} \\
y_{1}-y_{2}-\delta_{y} \\
\theta_{1}-\theta_{2} \\
\frac{1}{2}\left(x_{1}-x_{3}\right)^{2}+\frac{1}{2}\left(y_{1}-y_{3}\right)^{2}+\frac{1}{2}\left(\theta_{1}-\theta_{3}\right)^{2}-\delta
\end{array}\right]
$$

the codistribution $\mathrm{d} \mathcal{C}$ will be given by

$$
\begin{aligned}
& \mathbf{d} \mathcal{C}=\operatorname{span}\left\{\mathbf{d} x_{1}-\mathbf{d} x_{2}, \mathbf{d} y_{1}-\mathbf{d} y_{2}, \mathbf{d} \theta_{1}-\mathbf{d} \theta_{2},\right. \\
&\left(x_{1}-x_{3}\right) \mathbf{d} x_{1}+\left(y_{1}-y_{3}\right) \mathbf{d} y_{1}+\left(\theta_{3}-\theta_{1}\right) \mathbf{d} \theta_{1} \\
&\left.+\left(x_{3}-x_{1}\right) \mathbf{d} x_{3}+\left(y_{3}-y_{1}\right) \mathbf{d} y_{3}+\left(\theta_{1}-\theta_{3}\right) \mathbf{d} \theta_{3}\right\} .
\end{aligned}
$$

Combining $\mathbf{d} \mathcal{C}$ and $\Delta^{\perp}$ into $\Omega$ one easily verifies that $\operatorname{dim} \Omega$ is at maximum 7 since a basis for $\Omega$ is formed by the four forms spanning $\mathrm{d} \mathcal{C}$ and three more forms spanning $\Delta^{\perp}$. This means that this formation is indeed feasible since $\operatorname{dim} \Omega \leq 7<9=$ $m$. We can therefore conclude by Theorem 4.1 , that there are trajectories for each agent satisfying the formation constraints as well as its kinematics. In general we can have more forms than $m$ and still conclude feasibility since such forms may be linearly dependent. In the next section we will see how one can control the individual agents while maintaining the formation and gain some insight into the group trajectories.

\section{B. Group Abstraction}

Whenever more than one independent solution exist, the solution space of equation $\Omega \cdot X=0$ can be used to extract a lower dimensional control system that will preserve the formation along its trajectories. This new control system defined by the group distribution $G=\left\{X: \mathbb{R}^{m} \rightarrow \mathbb{R}^{m}: \omega \cdot X=0 \forall \omega \in\right.$ $\Omega\}$ is an abstraction that hides away low-level control necessary to maintain the formation, and can be used in higher levels of control. If we denote by $\left\{K_{1}, K_{2}, \ldots, K_{k}\right\}$ a basis for the kernel of $\Omega$, we can write the solution of Problem 3.3 as

$$
\dot{x}=\sum_{j=1}^{k} K_{j} w_{j}
$$

Since $\Omega \cdot K_{j}=0$ for any $j \in\{1,2, \ldots, k\}$ we conclude that for any input trajectory $w: I \rightarrow \mathbb{R}^{k}$, the corresponding state trajectory $x(t)$ satisfies $\mathcal{C}_{i j}^{p}(x(t))=0$ for all $\left(v_{i}, v_{j}\right) \in E$, $p=1,2, \ldots, \phi(i, j)$ and $t \in I$. The centralized nature of the problem is also reflected on the control abstraction. When one or more of the control inputs $w_{i}$ are used, interagent cooperation is necessary to implement the new direction of motion since each vector $K_{j}$ specifies the motion for all formation agents.

Example 4.3: We now return to the previous example and compute a basis for the kernel of $\Omega$. Straightforward computations lead to the following basis vector fields

$$
\begin{aligned}
& K_{1}=\left[\begin{array}{c}
\left(\theta_{3}-\theta_{1}\right) \cos \theta_{1} \\
\left(\theta_{3}-\theta_{1}\right) \sin \theta_{1} \\
0 \\
\left(\theta_{3}-\theta_{1}\right) \cos \theta_{1} \\
\left(\theta_{3}-\theta_{1}\right) \cos \theta_{1} \\
0 \\
0 \\
0 \\
\left(x_{1}-x_{3}\right) \cos \theta_{1}+\left(y_{1}-y_{3}\right) \sin \theta_{1}
\end{array}\right] \\
& K_{2}=\left[\begin{array}{c}
\left.\left(\left(x_{1}-x_{3}\right) \cos \theta_{3}+\left(y_{1}-y_{3}\right)\right) \sin \theta_{3}\right) \cos \theta_{1} \\
\left.\left(\left(x_{1}-x_{3}\right) \cos \theta_{3}+\left(y_{1}-y_{3}\right)\right) \sin \theta_{3}\right) \sin \theta_{1} \\
0 \\
\left.\left(\left(x_{1}-x_{3}\right) \cos \theta_{3}+\left(y_{1}-y_{3}\right)\right) \sin \theta_{3}\right) \cos \theta_{1} \\
\left.\left(\left(x_{1}-x_{3}\right) \cos \theta_{3}+\left(y_{1}-y_{3}\right)\right) \sin \theta_{3}\right) \sin \theta_{1} \\
0 \\
\left.\left(\left(x_{1}-x_{3}\right) \cos \theta_{1}+\left(y_{1}-y_{3}\right)\right) \sin \theta_{1}\right) \cos \theta_{3} \\
\left.\left(\left(x_{1}-x_{3}\right) \cos \theta_{1}+\left(y_{1}-y_{3}\right)\right) \sin \theta_{1}\right) \sin \theta_{3} \\
0
\end{array}\right] \\
& K_{3}=\left[\begin{array}{c}
\left(\theta_{1}-\theta_{3}\right) \cos \theta_{1} \\
\left(\theta_{1}-\theta_{3}\right) \sin \theta_{1} \\
\left(x_{1}-x_{3}\right) \cos \theta_{1}+\left(y_{1}-y_{3}\right) \sin \theta_{1} \\
\left(\theta_{1}-\theta_{3}\right) \cos \theta_{1} \\
\left(\theta_{1}-\theta_{3}\right) \cos \theta_{1} \\
\left(x_{1}-x_{3}\right) \cos \theta_{1}+\left(y_{1}-y_{3}\right) \sin \theta_{1} \\
0 \\
0 \\
0
\end{array}\right] .
\end{aligned}
$$

These vector fields define the control system

$$
\dot{x}=K_{1} w_{1}+K_{2} w_{2}+K_{3} w_{3} \text {. }
$$

To gain some insight on the trajectories of this control system, we display in Fig. 2 the formation evolution when the open loop control $w_{1}=1, w_{2}=0, w_{3}=0$ is used. The formation evolution is characterized by agent 3 rotating around some point while agent 1 and 2 perform straight line motions. The constraint between agents 1 and 2 is clearly satisfied since their motion is characterized by the same heading angle and a fixed distance between their positions. Not so obvious is to conclude satisfaction of the constraint between agent 1 and 3. Since the position of agent 3 is constant we conclude that the change in its orientation compensates the change in the distance between agent 1 and 3 in order for constraint (20) to be satisfied. When the formation 


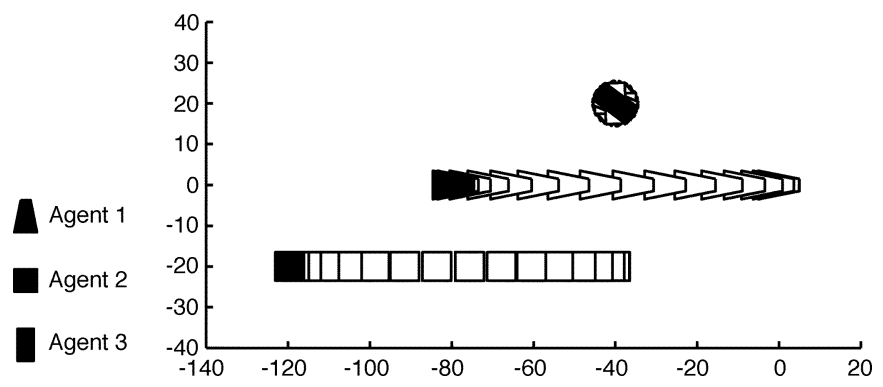

Fig. 2. Formation flow along vector field $K_{1}$.

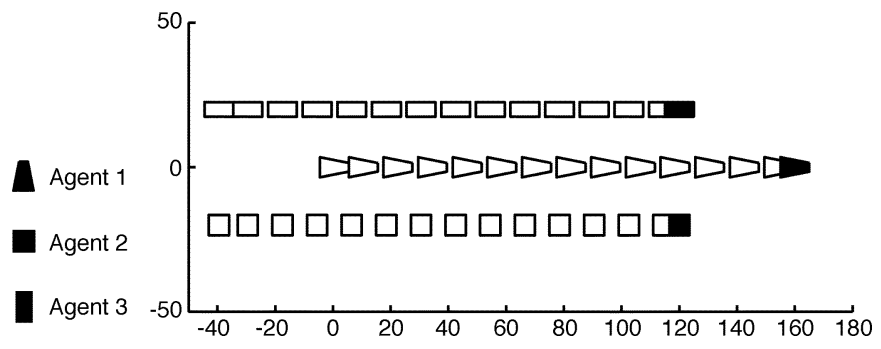

Fig. 3. Formation flow along vector field $K_{2}$.

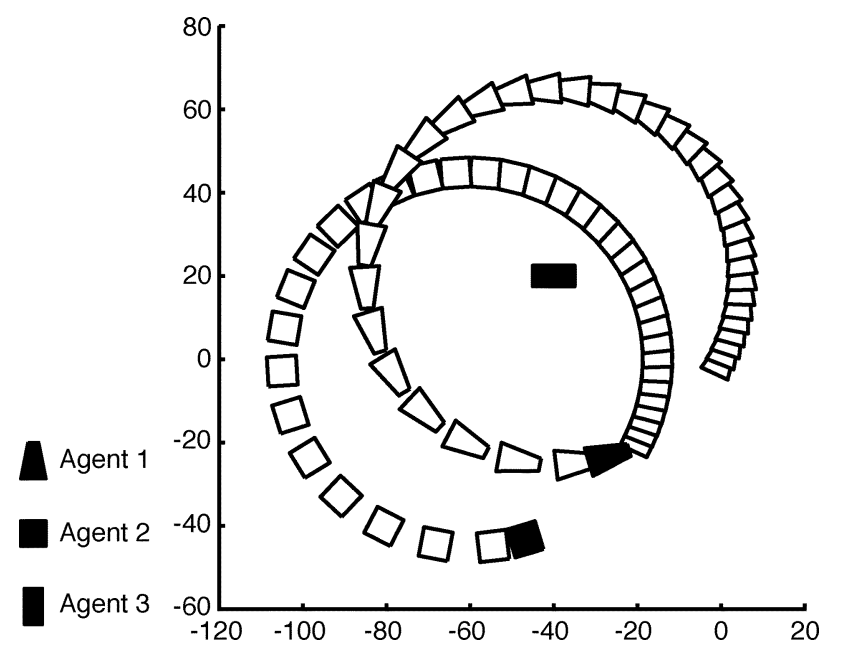

Fig. 4. Formation flow along vector field $K_{3}$.

flows along vector field $K_{2}$ corresponding to open loop control $w_{1}=0, w_{2}=1, w_{3}=0$, all the agents move along parallel trajectories as displayed in Fig. 3. This was achieved since their initial orientations were identical. When this is not the case, more complex motions characterize the flow along $K_{2}$. However, it is always possible to achieve identical orientations by flowing along $K_{1}$ or $K_{3}$. The formation flow along basis vector $K_{3}$ is somewhat dual to $K_{1}$. Instead of agent 1 rotating around himself to achieve different configuration errors regarding agent 1 , agent 3 is now stopped and the remaining agents revolve around it as suggested in Fig. 4. To generate more complex motions for the formation, other open or closed loop control laws can be used with the group abstraction (22).

\section{CONCLUSION}

In this paper we have proposed a framework to determine motion feasibility for multi-agent formations. Algebraic conditions were developed to determine motion feasibility for undirected formations. The abstraction problem was also addressed and solved by constructing a model of the formation as a whole, guaranteeing that the formation constraints are preserved along any of its trajectories. Although we have considered kinematic models, current research is focusing on the use of existing techniques such as backstepping [16] or kinematic reductions [6] to extend the presented results toward dynamical models for the agents.

\section{REFERENCES}

[1] R. Abraham et al., "Manifolds, tensor analysis and applications," in Applied Mathematical Sciences, New York: Springer-Verlag, 1988.

[2] T. Balch and R. Arkin, "Behavior-based formation control for multirobot systems," IEEE Trans. Robot. Autom., vol. 14, no. 6, pp. 926-939, Dec. 1998.

[3] R. W. Beard et al., "A coordination architecture for spacecraft formation control," IEEE Trans. Control Syst. Technol., vol. 9, no. 6, pp. 926-939, Nov. 2001.

[4] C. Belta and V. Kumar, "Motion generation for formations of robots: a geometric approach," in Proc. Int. Conf. Robotic Automation, Seoul, Korea, 2001, pp. 1245-1250.

[5] W. Blake and D. Multhopp, "Design, performance, and modeling considerations for close formation flight," presented at the AAIA Atmospheric fl;ight mecganics Conf. Exhibit, Boston, MA, Aug. 1998, Paper A98-3720410-08.

[6] F. Bullo and K. M. Lynch, "Kinematic controllability for decoupled trajectory planning in underactuated mechanical systems," IEEE Trans. Robot. Autom., vol. 17, no. 4, pp. 402-412, Aug. 2001.

[7] D. J. Cook, P. Gmytrasiewicz, and L. B. Holder, "Decision-theoretic cooperative sensor planning," IEEE Trans. Pattern Anal. Mach. Intell., vol. 18, no. 10, pp. 1013-1023, Oct. 1996.

[8] J. Cortes et al., "Coverage control for mobile sensing networks," in Proc. IEEE Conf. Robotic Automation, Arlington, VA, May 2002, pp. $1327-1332$.

[9] J. Desai et al., "Control of changes in formation of multi-robot teams," in Proc. Int. Conf. Robotic Automation, Detroit, MI, 1998, pp. 1556-1561.

[10] T. Eren et al., "A framework for maintaining formations based on rigidity," in Proc. 15th IFAC World Congr. Autom. Control, Barcelona, Spain, Jul. 2002, pp. 2752-2757.

[11] A. Isidori, Nonlinear Control Systems, 2nd ed, New York: SpringerVerlag, 1989.

[12] A. Jadbabaie et al., "Coordination of groups of mobile autonomous agents using nearest neighbor rules," IEEE Trans. Autom. Contr., vol. 8, no. 6, pp. 988-1001, Jun. 2003.

[13] N. E. Leonard and E. Fiorelli, "Virtual learders, artificial potentials and coordinated control of groups," in Proc. 40th IEEE Conf. Decision and Control, Orlando, FL, Dec. 2001, pp. 2968-2973.

[14] P. Ogren et al., "A control Lyapunov function approach to multi-agent coordination," IEEE Trans. Robot. Autom., vol. 18, no. 5, pp. 847-851, Oct. 2002.

[15] R. Olfati-Saber and R. M. Murray, "Distributed structural stabilization and tracking for formations of dynamics multi-agents," in Proc. 41st IEEE Conf. Decision and Control, Las Vegas, NV, Dec. 2002, pp. 209-215.

[16] R. Sepulchre et al., Constructive Nonlinear Control. New York: Springer-Verlag, Jan. 1997, Communications and Control Engineering.

[17] P. Tabuada et al., "Feasible formations of multi-agent systems," in Proc. American Control Conf., Arlington, VA, Jun. 2001, pp. 56-61.

[18] — "Decentralizing formations of multi-agent systems," presented at the 10th Mediterranean Conf. Control Automation, Lisbon, Portugal, Jul. 2002.

[19] H. G. Tanner et al., "Input to state stability on formation graphs," in Proc. 41st IEEE Conf. Decision and Control, Las Vegas, NV, Dec. 2002, CD-ROM, pp. 2439-2444.

[20] C. Tomlin et al., "Conflict resolution for air traffic management: a study in multi-agent hybrid systems," IEEE Trans. Autom. Contr., vol. 43, no. 4, pp. 509-521, Apr. 1998.

[21] P. Varaiya, "Smart cars on smart roads: problems of control," IEEE Trans. Autom. Contr., vol. 38, no. Feb., pp. 195-207, 1993. 


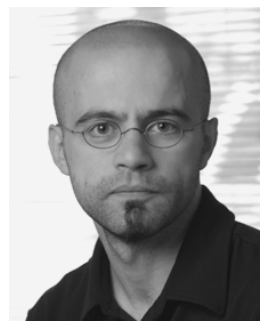

Paulo Tabuada (S'00-M'02) was born in Lisbon, Portugal, in 1975. He received the "Licenciatura" degree in aerospace engineering from Instituto Superior Tecnico, Lisbon, Portugal, in 1998 and the Ph.D. degree in electrical and computer engineering from the Institute for Systems and Robotics, a private research institute associated with Instituto Superior Tecnico in 2002.

From January 2002 to July 2003, he was a Postdoctoral Researcher at the University of Pennsylvania, Philadelphia. He is currently an Assistant Professor in the Department of Electrical Engineering, University of Notre Dame, Notre Dame, IN. His research interests include verification and control of discreteevent, timed, hybrid, embedded systems and networked systems as well as geometric control theory, categorical systems theory, Hamiltonian, hierarchical, and distributed control systems.

Dr. Tabuada was the recipient of the Francisco de Holanda prize in 1998 for the best research project with an artistic or aesthetic component awarded by the Portuguese Science Foundation. He was a finalist for the Best Student Paper Award at both the 2001 American Control Conference and the 2001 IEEE Conference on Decision and Control and he was an Outstanding Reviewer for the IEEE TRANSACTIONS ON AUTOMATIC CONTROL in 2002 and 2003.

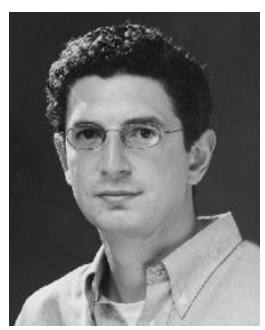

George J. Pappas (S'91-M'98) received the B.S. degree in computer and systems engineering and the M.S. degree in computer and systems engineering from Rensselaer Polytechnic Institute, Troy, NY, in 1991 and 1992, respectively. In 1994, he was a Graduate Fellow at the Division of Engineering Science of Harvard University. He received the Ph.D. degree from the Department of Electrical Engineering and Computer Sciences at the University of California at Berkeley, in 1998.

In 1999, he was a Postdoctoral Researcher at the University of California at Berkeley and the University of Pennsylvania, Philadelphia. In 2000, he joined the University of Pennsylvania as an Assistant Professor in the Department of Electrical and Systems Engineering, where he is currently an Associate Professor and the Graduate Group Chair. He also holds a secondary appointment in the Department of Computer and Information Sciences. He has co-edited the 2004 volume on Hybrid Systems: Computation and Control in the Lecture Notes in Computer Science Series (New York: Springer-Verlag, 2004). He has published over 100 articles in the areas of hybrid systems, hierarchical control systems, distributed control systems, nonlinear control systems, geometric control theory, with applications to flight management systems, robotics, and unmanned aerial vehicles.
Dr. Pappas is the recipient the NSF CAREER award in 2002 as well as the 2002 NSF Presidential Early Career Award for Scientists and Engineers (PECASE). He has received the 1999 Eliahu Jury Award for Excellence in Systems Research from the Department of Electrical Engineering and Computer Sciences at the University of California at Berkeley. His and his student's papers were finalists for the Best Student Paper Award at the 1998 IEEE Conference on Decision and Control, 2001 American Control Conference, 2001 IEEE Conference on Decision and Control, and 2004 American Control Conference.

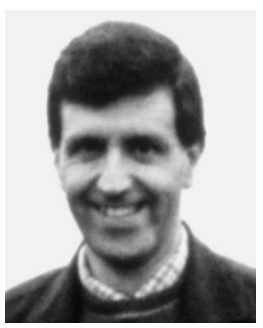

Pedro Lima (S'85-M'94) received the Ph.D. degree in electrical engineering from the Rensselaer Polytechnic Institute, Troy, NY, in 1994.

Currently, he is an Assistant Professor at Instituto Superior Técnico, Lisbon Technical University, Lisbon, Portugal. He is also a member of the Institute for Systems and Robotics, a Portuguese private research institution, where he is Co-Coordinator of the Intelligent Systems Group and a Member of the Scientific Council. His scientific interests are in the areas of hybrid systems, discrete event systems, and reinforcement learning, mainly in their applications to complex large-scale systems, such as multirobot systems. He is the author of one book and several journal and conference papers. He has also been very active in the promotion of science and technology to the society, through the organization of robotics events in Portugal, including the Portuguese Robotics Open since 2001.

Dr. Lima is a Trustee of the RoboCup Federation, and was the General Chair of RoboCup2004, held in Lisbon. He is a Member of the Editorial Board of the Portuguese magazine Robótica, a Member of the International Program Committee of several international conferences in robotics, as well as a Reviewer of several journals and international Ph.D. theses in the area. He is also the Co-Editor of two special issues of the IEEE Robotics and Automation Magazine, one Special Issue of Elsevier's Journal of Robotics Autonomous Systems. 